\title{
Polysèmes
}

Revue d'études intertextuelles et intermédiales

\section{Passages sans issue dans «The Aspern Papers » de Henry James}

Nelly Valtat-Comet

\section{(2) OpenEdition}

Journals

Édition électronique

URL : http://journals.openedition.org/polysemes/1604

DOI : 10.4000/polysemes.1604

ISSN : 2496-4212

Éditeur

SAIT

Édition imprimée

Date de publication : 1 janvier 2003

Pagination : 31-46

ISSN : 0999-4203

\section{Référence électronique}

Nelly Valtat-Comet, "Passages sans issue dans «The Aspern Papers » de Henry James », Polysèmes [En ligne], 6 | 2003, mis en ligne le 10 janvier 2017, consulté le 01 mai 2019. URL : http:// journals.openedition.org/polysemes/1604; DOI : 10.4000/polysemes.1604

Ce document a été généré automatiquement le 1 mai 2019.

Polysèmes 


\title{
Passages sans issue dans « The Aspern Papers » de Henry James
}

\author{
Nelly Valtat-Comet
}

1 Le récit intitulé "The Aspern Papers $»^{1}$ est paru en 1888 , c'est-à-dire au milieu chronologique presqu'exact de la carrière nouvellistique de James. Riche de cent treize œuvres courtes, cette carrière s'ouvre en 1864 avec «A Tragedy of Error » et se clôt en 1910 sur l'explicite circularité de « A Round of Visits ».

2 De fait, "The Aspern Papers» est le centre de convergence de nombreuses préoccupations thématiques et techniques jamesiennes. On y trouve rassemblés: la confrontation Europe/Amérique, puisque James a choisi de conférer à la figure byronienne de Jeffrey Aspern une peu vraisemblable nationalité américaine; la dichotomie vie/art, ou expérience/observation, accompagnée d'avatars tels que le voyeurisme; une mysogynie diffuse et la frayeur du protagoniste principal face à l'engagement amoureux; le thème du double avec le couple formé par un grand écrivain et son disciple inconditionnel; le rôle de la critique littéraire et son rapport avec la vie privée des auteurs ; la cristallisation des désirs autour de portraits ou de documents écrits perçus par certains personnages comme des substituts de personnes; la poursuite d'un secret dont ni le protagoniste, ni le lecteur, ne connaîtra finalement la teneur; l'exploitation de la maison et de la ville comme images du corps et de l'appareil psychique ; la communication avec les défunts et la nostalgie d'un passé idéalisé, proche mais révolu.

3 Au plan de la stratégie narrative, "The Aspern Papers" est avec "The Turn of the Screw » le plus connu et le plus analysé des récits jamesiens à la première personne, l'un des lieux d'expression de ce «je» dont James s'est détourné dans ses romans, et qu'il a caractérisé dans une préface comme étant « the darkest abyss of romance ».

4 James recourt par deux fois au mot italien « passegio » au cours de « The Aspern Papers ». Évoquant au bénéfice du narrateur et personnage principal ses années de jeunesse pour les orner d'un éclat qu'on devine pathétiquement illusoire, Miss Tita se souvient: «there was scarcely a week that they had not some visitor or did not make some delightful 
passegio in the city " (317). Le passegio de Miss Tita, ce sont les délices de la promenade, en gondole ou à pied, dans cette Venise romanesque et romantique où le narrateur succombera tant de fois au désir de se perdre. Plus tard, alors qu'approche le dénouement de l'histoire, le mot passegio revient, cette fois dans la bouche grossière et méprisante du valet, pour désigner le convoi funéraire, discret, presque furtif, de Juliana Bordereau: «But a funeral you could scarcely call it, signore; it was a dull little passegio of two gondolas » (365). De la promenade romantique à l'ultime voyage sur les eaux sombres d'un étroit canal, l'écho se fait lugubre et ironique, renforcé par le renversement de sens opéré pour l'autre mot commun aux deux phrases: le "scarcely» de Miss Tita qui signifiait l'insouciance et l'abondance ("there was scarcely a week...») et le "scarcely " de pénurie et d'oubli ( «a funeral you could scarcely call it») qui résonne aux côtés de l'autre occurrence du terme "passegio». Il nous semble qu'il y a là plus qu'une coquetterie de langage, plus que la récurrence d'un procédé mimétique visant par quelques italianismes (ou plutôt, ici, quelques « vénitianismes ») à étoffer le côté exotique de la mise en scène vénitienne.

5 Voici donc le «passage » mis en valeur, italianisé et italicisé, parmi les autres thèmes de la nouvelle. Nous examinerons la mise en fiction par le texte de James de deux acceptions de ce terme : le passage en tant qu'étape, lieu intermédiaire où l'on ne s'arrête que pour mieux repartir, et le passage comme brèche dans un obstacle, comme ouverture, comme trouée qui permet d'atteindre une destination auparavant inaccessible. Le mot anglais " passage » est présent, lui aussi, dans le texte. Paradoxalement, il désigne d'abord le grand salon d'apparat du palais Bordereau, et transforme ainsi en une sorte de corridor un lieu architectural normalement assigné au séjour et à la conversation. À la page 285 ce salon est " the grand, obscure vista », et le narrateur nomme " impenetrable regions » les autres pièces du même étage. Le grand salon occupe en effet un emplacement stratégique pour qui veut, comme le narrateur, explorer la maison, car il distribue l'accès aux différentes chambres et lui seul permet d'atteindre l'appartement interdit, celui de Juliana Bordereau. Lieu gothique par excellence, ce salon est voué au vide, au silence, à l'obscurité et aux iridescences quasi-fantomatiques. Il est d'abord la duplication architecturale, au premier étage, du grand hall d'entrée situé au rez-de-chaussée, et dont la vocation est bien le passage: "the wide, dusky, stone-paved passage which on the ground floor corresponded with our grand sala » (322). L'adjectif « dusky», repris ici, caractérise le grand salon lui-même à la page 284. Il s'accompagne au fil du texte d'autres adjectifs tels que « gloomy », «faded» (285), « tarnished », « dim » (276), « dark» (360), qui suggèrent une atmosphère mystérieuse et menaçante, où les rares lueurs ne sont que reflets sur le sol de marbre : «the bare scagliola floor gleamed vaguely » (284), « the hard shining floor » (285), et où le moindre bruit de pas se transforme en écho inquiétant. En ce lieu plus propice aux apparitions qu'aux entrées en scène, les deux femmes semblent toujours surgir d'un autre monde. C'est le lieu de la non-rencontre et de la noncommunication: toutes les conversations qui s'y déroulent ne font qu'épaissir l'incompréhension qui sépare les personnages.

6 Pour obscur qu'il soit, ce grand salon n'en est pas moins percé de vastes fenêtres qui s'ouvrent, pour peu qu'on se donne la peine d'écarter les persiennes, d'une part sur le jardin, d'autre part sur un balcon qui offre une vue du canal. C'est sur ce balcon que le narrateur/personnage opère auprès de Miss Tita l'une de ses attaques verbales les plus directes quant à l'existence et à la cachette des fameux papiers d'Aspern. Pour ce faire, il a ouvert une fenêtre, acte choquant dans une maison où les fenêtres, vues de l'extérieur, 
sont habituellement "as expressive as eyes consciously closed» (306). Puis les deux personnages sont sortis sur le balcon, un mouvement rendu dans le texte par le verbe " to pass out " («I opened the window and we passed out on the balcony », 356). L'ambiguité du jeu sur les deux sens de « pass out » ne fait que se confirmer lorsque la vue, au dehors, est décrite comme un sorte de duplication du salon : le canal est lui aussi un passage, lui aussi il est « hushed and void», les seules lueurs dans la nuit y sont elles aussi des reflets et des doubles: «A lamp, here and there, over the narrow black water, glimmered in double » (356). À relire ces lignes il nous vient à l'esprit la préface de The Portrait of a Lady, où dans une métaphore longuement filée James assimile l'art du récit de fiction à une maison percée d'innombrables fenêtres, toutes donnant sur le théâtre de la vie humaine². Si l'on veut appliquer au grand salon du palais Bordereau la même valeur métaphorique, si l'on veut voir dans l'acte d'ouvrir une fenêtre l'affirmation d'une volonté d'écriture et de représentation de la vie, alors les lignes que nous venons de commenter accusent l'échec du narrateur en tant qu'écrivain. Sa vision, son " point de vue " (pour reprendre les termes de James) ne débouchent sur rien, le corridor obscur et fantomatique n'ouvre ses fenêtres que sur un autre corridor obscur et fantomatique. James semble par là dénier à son personnage narrateur le droit de prétendre au statut d'artiste, ou du moins à celui d'artiste jamesien.

Ce relevé des occurrences du terme "passage » ou de ses équivalents dans le texte nous permet d'esquisser une réponse à la question des objectifs du passage en tant que thème présent dans la nouvelle. Vers qui, vers quoi, vers quelle destination cherche-t-on, dans "The Aspern Papers ", à se frayer un passage, à percer une brèche? Le narrateur anonyme, qui est aussi le personnage principal et qui s'exprime à la première personne, exerce le métier de critique littéraire. Il est le co-auteur d'une biographie du poète romantique et américain Jeffrey Aspern, inventé par James sur l'inspiration à la fois de Byron et de Shelley. L'objectif et l'obsession du protagoniste, exposés très clairement au début de la nouvelle, est de rédiger un certain chapitre manquant à la biographie de son idole, à propos d'une liaison qu'Aspern aurait eue, comme sa poésie le laisse entendre, avec Juliana Bordereau quelque soixante ans avant le moment de l'histoire, c'est-à-dire vers les années 1820. Nous qualifierons cet objectif d'explicite et primaire. Pour l'atteindre, le personnage s'est donné un objectif (que nous qualifierons d'accessoire ou secondaire), qui consiste à littéralement mettre la main sur les "papiers d'Aspern ", entendons les lettres d'amour que le poète a peut-être écrites, dont il pense que Juliana est toujours dépositaire.

8 L'objectif avoué du protagoniste est donc d'accomplir un acte d'écriture : il veut écrire une histoire d'amour, dont il ne serait pas le héros mais l'historien, le rapporteur et le commentateur, situation jamesienne par excellence. Cependant, même si la découverte et la lecture des hypothétiques "papiers» avaient lieu, elles ne seraient qu'un passage obligé, qu'une étape indispensable dans l'accomplissement de l'objectif primaire. Cet acte d'écriture-là, la rédaction du chapitre supplémentaire dans la biographie, aurait lieu semble-t-il hors l'histoire. C'est un objectif «supra-diégétique ». La fin de "The Aspern Papers" nous apprend l'échec de l'entreprise : Juliana Bordereau est morte sans avoir livré son secret; le narrateur n'a jamais pu lire, ni même entrevoir les papiers que Miss Tita affirme avoir brûlés, il n'écrira pas d'histoire d'amour, la biographie d'Aspern restera incomplète, et le protagoniste se transforme en chroniqueur de son propre parcours demeuré stérile. 
Ce parcours, il le met en scène dès les premières lignes du récit en choisissant des termes sémantiquement liés à la notion de déplacement, de progression physique, topographique. Sans Mrs. Prest, dit-il, Mrs. Prest étant le personnage, récurrent dans les œuvres de James, de la femme du monde américaine installée dans une ville d'Europe et veillant au bien-être et à la perpétuation du microcosme formé par ses compatriotes (c'est l'équivalent de Mrs. Walker dans "Daisy Miller»); sans Mrs. Prest, dit le protagoniste, «I should have made but little advance » (275), «It was she », poursuit-il, " who invented the short-cut, who severed the Gordian knot ».

10 Cette introduction appelle plusieurs remarques. D'abord, nous apprenons qu'il est question d'un cheminement avant même d'en connaître la destination, ce qui peut suggérer que l'un est plus important que l'autre. Ensuite, nous imaginons que cette destination a fini par être atteinte, puisque Mrs. Prest a bien prêté son indispensable concours. Une première lecture nous fait semble-t-il entrevoir un narrateur usant du privilège de celui qui connaît la fin de l'histoire, et aiguisant notre intérêt à l'aide de prolepses à peine déguisées. Enfin, la mention du nœud gordien, outre qu'elle ancre le récit dans un système de références mythologiques et religieuses où cohabiteront par la suite Orphée et les Ménades, Satan et la Vierge Marie, porte une connotation de liberté, de voie ouverte vers toutes les conquêtes. En même temps, avec l'idée de "dénouer », de « dénouement », elle nous projette, elle aussi, vers la fin de l'histoire. Si cette histoire est la représentation d'un cheminement, et que sa fin est contenue dans son début, James ne nous propose-t-il pas d'emblée un pacte de lecture présupposant justement l'inverse de l'idée d'avancée, une sorte de retour au point initial ? Par ailleurs, le terme " advance » et l'allusion à Alexandre le Grand inaugurent une autre veine métaphorique, présente tout au long de l'œuvre et liée à la notion de progression : c'est celle de la guerre de conquête. Il sera question plus tard, et dans l'ordre, de siège, de plan de campagne, de lutte, de champ de bataille, de citadelle, de bombardement et de la reddition espérée de l'adversaire.

11 Les deux premières phrases de la nouvelle créent donc un contexte métaphorique à la fois de progression et de conquête, mais nous ne tardons pas à apprendre qu'il s'agit à plus proprement parler d'une quête, la quête, comme souvent chez James, d'un objet à la fois précieux, mystérieux et absent, ici les fameux "papiers » qui, pour le protagoniste, semblent receler "la réponse à l'énigme de l'univers" (277), de même qu'il considère Aspern comme un dieu. Pour arriver jusqu'à ces papiers (dans le texte « to arrive at the papers ", 282), le biographe se propose de s'assurer de la complicité de Miss Tita. Pour cela, il adopte avec elle une attitude et dans sa narration un langage qui donnent à penser, à elle comme au lecteur, qu'il lui fait la cour. Nous assistons à un détournement de la veine métaphorique militaire, qui exprime l'intention du biographe de forcer non seulement l'entrée du palais, mais aussi celle du cœur de Miss Tita. Le langage de la conquête assume alors des connotations d'amour courtois qui ont été analysées avec pertinence dans d'autres études.

12 S'assurer de la bienveillance de Miss Tita consitue une première étape intermédiaire en direction des papiers. Le cheminement métaphorique se développe autour de trois contenus de sens. Le premier se résume en une parodie de l'itinéraire sentimental figuré par la carte du Tendre. Ici l'usage de la métaphore se démultiplie, puisque "l'avancée » dont il est question dans la nouvelle ne figure pas directement la démarche amoureuse : elle figure le code de l'amour courtois, qui lui-même représente métaphoriquement la démarche amoureuse. 
13 Le deuxième contenu de sens affecté à la métaphore du cheminement se veut quant à lui concret, géographique et horizontal ; il concerne l'itinéraire qui mène de la gondole de Mrs. Prest à l'intérieur du palais des demoiselles Bordereau, et à l'intérieur de ce palais, du grand salon d'apparat au parloir de Juliana, et du parloir à la chambre, et dans la chambre à la cachette qu'il faudra découvrir, où ne manqueront pas de se trouver les documents.

14 En troisième lieu, et ici la notion de cheminement revêt un sens beaucoup plus abstrait que nous qualifierons de vertical, le protagoniste cherche à traverser le temps, qu'il spatialise en le nommant « distance of time » (278). Il cherche à percer une brèche dans la frontière entre présence et absence, entre vie et mort, à se frayer un passage afin d'atteindre ce qu'il nomme la « vérité » de Jeffrey Aspern. Témoins les nombreux emplois d'expressions telles que « to bridge the gulf of time » (282), « to go back» (306), « to rake up the past ", "to dig" (341), " to reach", et même " to overreach », ou duper pour atteindre (304).

Cette volonté dévoile la présence d'un deuxième objectif avoué dans la démarche du biographe, un objectif corollaire du premier, mais qui déborde des intentions purement historiques et biographiques. Ce qu'il cherche à faire, c'est littéralement entrer en contact avec le défunt dans toutes les dimensions de sa personne: non seulement avec le poète, mais aussi avec le maitre à penser capable de conseiller dans les situations les plus épineuses, avec l'amoureux doté d'un visage, d'un regard, d'une voix, d'une main que l'on peut étreindre, d'une peau que l'on peut caresser.

16 Le biographe tentera d'établir l'impossible contact par divers moyens, le plus évident étant une sorte de transitivité, par laquelle il s'imagine qu'un contact physique direct avec une personne qui aurait connu Aspern (en l'occurrence Juliana Bordereau), lui permettrait à lui, biographe, de participer à cette connaissance autrement que par les textes. Tout au long de son aventure il cherche, vainement, à s'approcher de Juliana, qui lui refuse avec persistance la moindre poignée de main, et même le moindre regard, puisqu'elle porte en permanence un voile. À défaut de Juliana, la transitivité se compliquant d'une étape, c'est à travers Tita qu'il tente de trouver, sinon Aspern, du moins une atmosphère, une époque que le grand homme aurait connue. Des deux femmes, il dit «they made my life continuous, in a fashion, with the illustrious life they had touched at the other end. I lost myself in this satisfaction to the point of assumingin my quiet extravagance-that poor Miss Tita also went back, went back, as I used to phrase it » (306). Continuité temporelle, contact physique, retour vers le passé ne sont que rêve où l'on se perd. Même dans ses moments de plus grand optimisme, le biographe reste conscient de l'absurdité de ce désir, et ne prend jamais ses illusions pour des réalités. La présence imaginée d'Aspern, ou même de son fantôme, n'est jamais rendue autrement qu'assortie de précautions verbales, comme en témoigne la série de « seemed » et de « it was as if » dans l'extrait suivant :

That spirit kept me perpetual company and seemed to look out at me from the revived immortal face-on which all his genius shone-of the great poet who was my prompter. I had invoked him and he had come; he hovered before me half the time; it was as if his bright ghost had returned to earth. It was as if he had said... (305)

17 La miniature représentant Aspern constitue l'autre point de contact envisagé. C'est une sorte de substitut des "papiers », que Juliana accepte de marchander et auquel se réduira finalement l'unique butin du chercheur. Cependant, lui non plus ne permet pas autre 
chose qu'une illusion de communication. Lorsque, dans un moment de profond désarroi où il refuse toujours de comprendre ce que Miss Tita attend de lui, le biographe appelle, en quelque sorte, le portrait à son secours, celui-ci demeure inerte et muet :

I only privately consulted Jeffrey Aspern's delightful eyes with my own (they were so young and brilliant, and yet so wise, so full of vision); I asked him what on earth was the matter with Miss Tita. He seemed to smile at me in friendly mockery, as if he were amused at my case... He was unsatisfactory, for the only moment since I had known him. (372)

Certes cette recherche avouée de contact avec l'au-delà n'a rien que de très banal dans l'univers jamesien. Il existe même une autre nouvelle, parmi les 112 écrites par James, qui narre le même type de démarche d'un biographe envers le défunt écrivain qu'il admire, il s'agit de "The Real Right Thing ", publiée en 1899. Or dans cette autre histoire, plusieurs éléments de la situation de "The Aspern Papers » sont inversés : c'est l'artiste défunt qui fait une irruption défensive dans la vie du biographe alors que celui-ci jouit du libre accès à ses papiers intimes grâce à une relation privilégiée avec la veuve. Le revenant interpose entre sa vie privée et la publication de celle-ci la masse opaque d'une présence fantomatique effrayante qui dépouille finalement le biographe de toute velléité de mener à bien son entreprise. Les deux histoires se résolvent par l'échec de l'écriture biographique, et l'avènement du récit de cet échec. Il est légitime de lire «The Real Right Thing ", récit par ailleurs souvent estimé sans grand intérêt, comme une mise au point adressée directement par Henry James à ses lecteurs. Il y opère un retour sur les thèmes, les situations et les personnages de "The Aspern Papers", il réécrit la nouvelle en inversant tous ses éléments principaux et démontre que, quelle que soit la manière dont on aborde ce sujet, l'aboutissement en est inéluctable.

Compléter la biographie d'Aspern, établir un contact avec lui : les deux objectif avoués s'avèrent l'un et l'autre inatteignables et la quête du narrateur se solde par un échec. Il est cependant un troisième objectif qui, bien qu'il affleure fréquemment dans le discours, ne figure pas dans les intentions exposées délibérément par le narrateur. De nombreux indices textuels donnent à penser que le narrateur cherche bien plus qu'un simple contact avec Aspern: il cherche une fusion, il cherche à devenir Aspern à la place d'Aspern, il veut lui-même être amoureux et poète. Jamais avoué, et même plusieurs fois dénié, ce désir transparaît dans le texte comme à l'insu du narrateur, jusqu'à prendre la forme de lapsus. L'un d'entre eux est détectable dans notre dernière citation. Lorsqu'on lit «I consulted Jeffrey Aspern's eyes with my own (they were so young and brilliant, and yet so wise, so full of vision) » (372), on ne sait plus très bien qui, d'Aspern ou du narrateur, a le regard jeune, vif et intelligent.

Parmi les nombreuses manifestations de ce désir de fusion, figure le choix du narrateur d'établir un code de communication avec ses lecteurs au moyen d'allusions mythologiques, artistiques, cuturelles et religieuses. D'abord ce narrateur se présente bien comme un narrateur écrivant. En tant que personnage il écrit, dit-il, une biographie d'Aspern. Mais le récit de sa propre histoire, il l'opère également par écrit, bien qu'il ne soit nulle part question du type de support choisi : ici, contrairement à bien d'autres nouvelles de James écrites à la première personne, pas de cahier, pas de lettre, pas de vieux papiers retrouvés par un ami, mais ce narrateur écrit, c'est indéniable, et il adopte à plusieurs reprises une attitude réflexive quant à sa méthode de narration: «I shall not take up space ", déclare-t-il par exemple à la page 278 , « with attempting to explain ». Ce récit écrit n'est pas destiné à rester secret, car il pose l'existence d'un narrataire non 
identifié : "You may imagine whether it cleared up the puzzle ( (343). Le récit est donc bien présenté comme une forme de communication avec ses éventuels lecteurs, des initiés avec qui le narrateur semble partager une culture fondée sur la connaissance des mythes antiques et celle de la ville de Venise, dans ses dimensions spatiales et historiques.

Revenons au nœud gordien de l'incipit. Nous savons qu'il induit d'emblée le lecteur en erreur, puisqu'il promet une conquête qui ne se fera pas. Ce n'est pas le biographe luimême qui a métaphoriquement tranché le nœud avant le début de l'histoire, mais Mrs. Prest, c'est-à-dire le personnage secondaire hautement artificiel (n'osons pas parler de personnage-ficelle lorsqu'il s'agit de nœud!). Le biographe n'est donc Alexandre le Grand que par procuration, et il ne réussira pas à s'extirper de cette situation de retrait en tant que sujet, puisque tout au long de la nouvelle les désirs, les sentiments, les souffrances, les conquêtes et toute la vie amoureuse n'existent pour lui qu'au travers essentiellement de Jeffrey Aspern, ou plutôt de ce qu'il imagine de Jeffrey Aspern.

Il idéalise d'abord Aspern comme son dieu, en l'affectant d'une avalanche de superlatifs : " one of the greatest names» (275), " one of the most genial men and one of the handsomest " (277), «that voice was one of the sweetest ever heard» (278). Puis, s'appliquant à réfuter certaines rumeurs selon lesquelles Aspern n'aurait pas toujours agi en parfait gentleman avec ses conquêtes féminines, il le disculpe en l'assimilant à Orphée poursuivi et massacré par les Ménades :

'Orpheus and the Maenads!' was the exclamation that rose to my lips when I first turned over his correspondence. Almost all the Maenads were unreasonable, and many of them insupportable; it struck me in short that he was kinder, more considerate than, in his place (if I could imagine myself in such a place !) I should have been. (278)

La référence au mythe d'Orphée doit être examinée ici dans ses multiples implications. Orphée, ayant reçu d'Apollon la lyre à sept cordes, lui en ajouta deux. Il atteignit ainsi le nombre de neuf, qui est aussi celui des muses et, peut-être par autre chose qu'une coïncidence, celui des chapitres qui rythment «The Aspern Papers ». Père mythique de tous les poètes grâce à l'invention de cette lyre à neuf cordes, Orphée est aussi un grand voyageur et la figure emblématique du passage du monde des vivants à celui des morts. Amoureux d'une unique femme, il entretient avec toutes les autres des relations ambiguës, car même s'il les méprise, il n'en est pas moins l'objet de leurs passions.

Les ramifications du mythe d'Orphée sont multiples dans l'histoire que nous conte le narrateur de "The Aspern Papers ». C'est explicitement Aspern qui est assimilé au père des poètes. Aspern possédait, nous dit-on, le charme d'une voix exquise : "one of the sweetest ever heard» (278). On retrouve l'élément liquide et le voyage, d'une part dans les allées et venues transatlantiques d'Aspern (d'après ce qu'en a compris son biographe il aurait effectué un retour en Europe pour retrouver Juliana, avant de la quitter définitivement et de s'installer en Amérique), d'autre part dans toutes les exploitations de la nature aquatique de Venise. Le passage dans le monde des morts est évidemment, en revanche, l'ambition du biographe pour lui-même, et c'est une première appropriation abusive du mythe, puisqu'il n'est pas le poète. Tout au plus réussira-t-il à se donner l'illusion que c'est Aspern qui vient à lui sous forme d'apparition surnaturelle. Le seul personnage en définitive qui assure une liaison, à la fois concrète et métaphorique, entre le monde des morts et celui des vivants, et cette fois le mythe subit un retournement, c'est Juliana Bordereau. Elle est l'amoureuse qui, comme Orphée, a rompu tout lien avec 
son temps depuis qu'elle a perdu l'objet de son amour, celle qui, bien que vivante dans le présent, dissimule son regard à la lumière du jour. Beaucoup d'interprétations se sont exprimées quant au masque effrayant que Juliana ne quitte jamais, nous y ajouterons celle qui consiste à y voir un parallélisme supplémentaire avec le mythe d'Orphée. Au point culminant de l'histoire le biographe, s'étant frayé un chemin dans l'obscurité vers la chambre de Juliana malade, s'apprête à forcer le secrétaire où il pense qu'elle a dissimulé les papiers. Juliana apparaît alors derrière lui, dévoilant son regard pour la première fois, et c'est à cet instant précis que le récit bascule vers sa catastrophe : la mort de Juliana et la perte irrémédiable des documents. Juliana est en effet celle qui effectue littéralement le passage vers le règne des morts, celle qui s'éloigne solitaire sur une embarcation au gré d'un canal qui ne peut que rappeler le Styx. On ne sait donc pas si Jeffrey Aspern était Orphée, mais on sait en revanche que le narrateur, lui, ne l'est pas.

Par une deuxième strate de codification culturelle, le narrateur sollicite la complicité des destinataires de son récit dans ses évocations du passé. Une série de noms de personnages historiques vient étoffer ce que le narrateur nomme « distance of time » (278): ce sont les allusions à Mrs. Siddons, à la Reine Caroline et à Lady Hamilton (277), ou encore à Casanova (318), qui situent cette "old Venice", vers laquelle le narrateur se sent tellement attiré, dans un contexte sulfureux d'amours libres et illicites. Ce clin d'œil au lecteur se retourne ironiquement contre le personnage qui, bien qu'il s'y intéresse de près, n'entend visiblement rien à une vie amoureuse mouvementée.

L'assimilation implicite de Jeffrey Aspern à Byron, détaillée par Adeline Tintner dans son article intitulé "Henry James and Byron, Victorian Romantic Relationship ", contredit par transitivité la nature divine que le narrateur affecte au personnage d'Aspern. «The Satanic Lord", en effet, tout autant que ses personnages fictifs, est connu pour sa cruauté envers les femmes. Or dans « The Aspern Papers ", c'est à lui-même et non pas à son idole que le biographe confère un aspect satanique : «I am prepared to roast all summer-as well as hereafter » (283), déclare-t-il à Mrs. Prest en manière de plaisanterie. Plus tard, il se représente dans le décor paradisiaque du jardin, occupé à provoquer la tentation de l'amour chez Miss Tita, en des scènes qui ne laissent pas de faire penser au Satan de Milton, dans Paradise Lost. Or le personnage échoue en tant que démon, puisque Miss Tita finalement ne cédera pas à ses discours, de la même manière qu'il échoue en tant qu'historien et biographe.

27 S'il n'est pas Orphée, le narrateur de "The Aspern Papers » n'est certes pas non plus Byron. Tout fonctionne comme s'il ne maitrisait pas le code qu'il emploie, comme s'il le laissait, sans s'en rendre compte, se retourner contre lui. La transmission de renseignements par l'intermédiaire de références culturelles s'opère semble-t-il directement de l'auteur au lecteur, à l'insu d'un narrateur trahi par son propre langage. Au-delà de l'impossible retour vers le passé, de l'impossible fusion avec une idole disparue, de l'impraticable indiscrétion biographique, cette impasse-là s'avère peut-être le plus invalidant des pièges pour un narrateur jamesien qui dit «je », car la réussite de "The Aspern Papers» repose sur la conjugaison des échecs de l'histoire et de son écriture. 


\section{BIBLIOGRAPHIE}

Bell, B.C. “Beyond irony in Henry James: 'The Aspern Papers'”. Studies in the Novel, 1981 Fall. Vol. 13 (3): 282-293.

Bell, M. “'The Aspern Papers': the unvisitable past ». The Henry James Review, 1989 Spring. Vol. 10 (2): 120-126.

Carton, E. “The Anxiety of Effluence: Criticism, Currency and 'The Aspern Papers”'. The Henry James Review 1989 Spring. Vol. 10 (2): 116-119.

Franklin, R.F. "Military metaphor and the organic structure of Henry James's 'The Aspern Papers"”. Arizona Quarterly, 1976. Vol. 32: 327-340.

Gargano, J.W. “'The Aspern Papers': the untold story”. Studies in Short Fiction, 1973. Vol. 10: 1-10. Jensen-Osinki, B. "The key to the palpable past: Miss Tina in 'The Aspern Papers"'. The Henry James Review, 1981 Fall. Vol. 3 (1): 4-10.

Oliveira, B. de. "Carlos Fuentes and Henry James: the sense of the past". Arizona Quarterly, 1981 Autumn. Vol. 37 (3): 237-244.

Perosa, S. “Henry James's ‘The Aspern Papers'”. Criticism: a Quarterly for Literature and the Arts, 1987 Fall. Vol. 29 (4): 125-133.

Rivkin, J. “Speaking with the dead: ethics and representation in 'The Aspern Papers"”. The Henry James Review, 1989 Spring. Vol. 10 (2): 135-141.

Rodgers, P.C. Jr. “Motive, agency and act in James's ‘The Aspern Papers”'. South Atlantic Quarterly, 1974. Vol. 73: 377-387.

Schneider, B.J. “The unreliable narrator: James's 'The Aspern Papers' and the reading of fiction”. Studies in Short Fiction, 1976. Vol. 13: 43-49.

Stocking, M.K. "Miss Tina and Miss Plin: the papers behind 'The Aspern Papers'. » The Evidence of the imagination: Studies of interactions between life and art in English Romantic Literature. D.H. Reiman, H. Donald, M.C. Jaye, C. Michael, B.T. Bennett (ed). New York: New York UP, 1978, 372-384

Tintner, A. R. "Henry James and Byron: a Victorian Romantic Relationship" The Byron Journal, 1981. Vol. 9: 52-63.

Waldmeier, J.J. “Miss Tina did it: a fresh look at 'The Aspern Papers"'. The Centennial Review, 1982 Summer. Vol. 26 (3): 256-267.

\section{NOTES}

1. The Complete Tales of Henry James, ed. Leon Edel, vol. 6, 275-382. Philadelphia and New York: J.B. Lippincott Company, 1963.

2. The Art of the Novel, 46 . 
INDEX

oeuvrecitee Aspern Papers (The) 